\title{
Heterogeneity Signs on Noncontrast Computed Tomography Predict Hematoma Expansion after Intracerebral Hemorrhage: A Meta-Analysis
}

\author{
Danfeng Zhang $\mathbb{D}^{1},{ }^{1}$ Jigang Chen, ${ }^{1}$ Qiang Xue $\mathbb{D}^{1},{ }^{1}$ Bingying Du $\left(\mathbb{D},{ }^{2}\right.$ Ya Li $\mathbb{D},{ }^{1}$ Tao Chen $\mathbb{D}{ }^{3}$ \\ Ying Jiang, ${ }^{1}$ Lijun Hou $\mathbb{D},{ }^{1}$ Yan Dong $\mathbb{D}^{1},{ }^{1}$ and Junyu Wang $\mathbb{D}^{1}$ \\ ${ }^{1}$ Department of Neurosurgery, Changzheng Hospital, Second Military Medical University, Shanghai 200003, China \\ ${ }^{2}$ Department of Neurology, Changhai Hospital, Second Millitary Medical University, Shanghai, China \\ ${ }^{3}$ Force 71571, PLA, China
}

Correspondence should be addressed to Lijun Hou; lijunhou@smmu.edu.cn, Yan Dong; yandongsmmul@163.com, and Junyu Wang; jerome2008@163.com

Received 23 September 2017; Accepted 5 December 2017; Published 10 January 2018

Academic Editor: Robert M. Starke

Copyright (C) 2018 Danfeng Zhang et al. This is an open access article distributed under the Creative Commons Attribution License, which permits unrestricted use, distribution, and reproduction in any medium, provided the original work is properly cited.

Background and Purpose. Hematoma expansion (HE) is related to clinical deterioration after intracerebral hemorrhage (ICH) and noncontrast computed tomography (NCCT) signs are indicated as predictors for HE but with inconsistent conclusions. We aim to clarify the correlations of NCCT heterogeneity signs with HE by meta-analysis of related studies. Methods. PubMed, Embase, and Cochrane library were searched for eligible studies exploring the relationships between NCCT heterogeneity signs (hypodensity, mixed density, swirl sign, blend sign, and black hole sign) and HE. Poor outcome and mortality were considered as secondary outcomes. Odds ratio (OR) and its $95 \%$ confidence intervals (CIs) were selected as the effect size and combined using random effects model. Results. Fourteen studies were included, involving 3240 participants and 435 HEs. The summary results suggested statistically significant correlations of heterogeneity signs with HE (OR, 5.17; 95\% CI, 3.72-7.19, $P<0.001$ ), poor outcome (OR, 3.60; 95\% CI, 1.98-6.54, $P<0.001$ ), and mortality (OR, 4.64; 95\%, 2.96-7.27, $P<0.001)$. Conclusions. Our findings suggested that hematoma heterogeneity signs on NCCT were positively associated with the increased risk of HE, poor outcome, and mortality rate in $\mathrm{ICH}$.

\section{Introduction}

Intracerebral hemorrhage (ICH) accounts for nearly $15 \%$ of the stroke and is usually regarded as the most deadly type $[1,2]$. It carries a poor prognosis, with $40 \%$ mortality at one month and only $20 \%$ survivors being independent at six months [3]. Initial volume, shape, and location of hematoma are reliable predictors of mortality and clinical functional outcomes $[1,4]$. However, these are unmodifiable factors on admission. Hematoma expansion (HE) is usually considered as hemorrhage volume growth of more than $12.5 \mathrm{~mL}$ or $33 \%$ on repeated head computed tomography (CT) and occurs in almost one-third of ICH patients [5]. As a modifiable factor, it has been regarded as an independent predictor for unpleasant outcomes and mortality after ICH [6-8].
The spot sign, an area of contrast enhancement visible on computed tomographic angiography (CTA), is a wellestablished factor which predicts HE in ICH patients [9, $10]$, and therefore it carries potential clinical implications in identifying patients at a high risk of early death and poor functional outcomes. However, identification of spot sign needs a CTA, which is much more expensive and timeconsuming than regular CT scan and not routinely conducted at acute phase in many clinical settings.

In contrast, as a preferred imaging test for ICH patients, noncontrast computed tomography (NCCT) is widely available due to its low price and convenience. Previous studies have examined the accuracy of NCCT signs such as hypodensity, mixed density, swirl sign, blend sign, and black hole sign in predicting $\mathrm{HE}$, but these conclusions are inconsistent 
based on different methods and subjects [11-16]. A better understanding of NCCT signs might help identify patients with stratified needs and potentially improve prognosis. We carry out this study to evaluate the correlations of NCCT heterogeneity signs with $\mathrm{HE}$ after ICH.

\section{Methods}

2.1. Search Strategy. Our meta-analysis was reported according to the recommendations of Preferred Reporting Items for Systematic Reviews and Meta-Analysis: The PRISMA Statement [17]. The protocol was not previously registered. Two authors (D.F. Z. and J.G. C.) searched PubMed, Embase, and Cochrane library independently for eligible studies exploring the relationships between heterogeneity signs on NCCT and HE on July 15th 2017 with no date limits. The language was limited to English. Terms "hypodensity", "mixed density", "swirl sign", "blend sign", and "black hole sign" were combined with free words "intracerebral hemorrhage" and "computed tomography" in retrieval. The search strategy for PubMed was available in online-only Data Supplement. The references of included articles were also examined.

2.2. Heterogeneity Signs and Endpoints. Available heterogeneity signs included in our study were hypodensity, mixed density, swirl sign, blend sign, and black hole sign on NCCT. According to previous studies, hypodensity, mixed density, and swirl sign on NCCT were defined as hypoattenuated or isoattenuated region(s) within hyperattenuated $\mathrm{ICH}$, while blend sign or black hole sign was considered as the combination of relatively hypoattenuating region and hyperattenuating area with a well-defined margin [16, 19-23]. All these definitions indicated hypoattenuated region(s) within hyperattenuated $\mathrm{ICH}$, and thus we used terms heterogeneity signs to represent hypodensity, mixed density, swirl sign, blend sign, and black hole sign on NCCT in current study.

Our endpoints were considered to be primary and secondary. The primary endpoint was $\mathrm{HE}$ and defined as a hematoma growth of " $>6 \mathrm{~mL}$ or $>33 \%$ " or " $>12.5 \mathrm{~mL}$ or $>33 \%$." The secondary endpoints were poor outcome and mortality (in-hospital or 90-day mortality). Poor outcome was defined as Glasgow Outcome Scale (GOS) score of 1 to 3 , or modified Rankin scale (mRS) score of more than 3, or Glasgow Come Scale (GCS) score of less than 8, or decreased GCS score of more than 3 .

2.3. Inclusion Criteria. Studies were included in our research if they (1) were case control or cohort studies; (2) recruited patients suffering ICH; (3) examined the associations between the presence of heterogeneity signs and primary or secondary endpoints. Studies with incomplete data were excluded and in the evaluation of heterogeneity signs; we considered hypodensity as the exposure factor if several NCCT signs were available in one study.

2.4. Data Extraction and Quality Assessment. Characteristics and data were extracted from included studies independently by three investigators (D.F. Z., J.G. C., and Y. L.), which were as follows: first author, year of publication, study design, study population, sample size, age and gender of subjects, time interval from ICH onset to CT, baseline GCS scores, definitions of heterogeneity signs and endpoints, and number of participants with or without events in heterogeneitysigns-positive and negative groups. Two authors (T. C., Y. D.) independently assessed methodological quality using the Newcastle-Ottawa Scale. Discrepancies among investigators were resolved by joint review.

2.5. Statistical Analysis. Dichotomous data were available for the meta-analysis. Odds ratio (OR) and its 95\% confidence intervals (CIs) were selected as the effect size. Heterogeneity among studies was assessed with the $I^{2}$ statistic and Chisquare test. $I^{2}$ values of $25 \%, 50 \%$, and $75 \%$ were considered as low, moderate, and high, respectively [24]. Random effects model was employed in all quantitative analysis. Subgroup analyses were conducted according to study design, sample size, mechanism of ICH, and time interval from symptom onset to CT. Sensitivity analyses were conducted by removing one study at a time. Publication bias was assessed with Egger's test. A significant level of $P=0.1$ was used in the evaluation of heterogeneity and publication bias. In other cases, $P$ value of less than 0.05 was deemed as significant. All statistical analyses were conducted using STATA software (version 12.0; Stata Corporation, College Station, TX).

\section{Results}

3.1. Literature Search. The study-retrieval process was shown in Figure 1. The initial search produced 966 studies from Embase, 434 studies from PubMed and 74 studies from Cochrane library. After the removal of duplicates and irrelated publications, 27 studies were potentially related to our review and the full texts were assessed. Finally, a total of 14 articles were included in our analysis. The review of reference lists of included studies yielded no eligible literatures.

3.2. Study Characteristics. Fourteen studies were included in the meta-analysis, which consisted of one prospective cohort study, three retrospective cohort studies, and ten case control studies, involving 3240 participants and $435 \mathrm{HEs}$ (Table 1 and Supplementary Table I). Five studies were conducted in North America $[11,15,21,25,26]$, four in Europe [13, 19, $22,27]$, and five in Asia [16, 20, 23, 28, 29]. Generally, the gender ratio was balanced except in Pruthi et al., where males account for $91.7 \%$ of sample size. Presence of hypodensities was reported in two studies [21, 26], with mixed density reported in two studies $[15,16]$, swirl sign in six studies $[11,13,19,21,25,27]$, blend sign in four studies [20-22, 30], and black hole sign in two studies $[23,28]$. One study reported several NCCT signs for the same cohort [21]. Definitions of heterogeneity signs and quality assessment of included studies were demonstrated in Tables I and II in the onlineonly Data Supplement.

3.3. Heterogeneity Signs and HE. Six studies were available to investigate the relationship between NCCT heterogeneity signs and $\mathrm{HE}[20,21,23,25,28,29]$. The summary OR suggested statistically significant relationship between 
PRISMA 2009 flow diagram

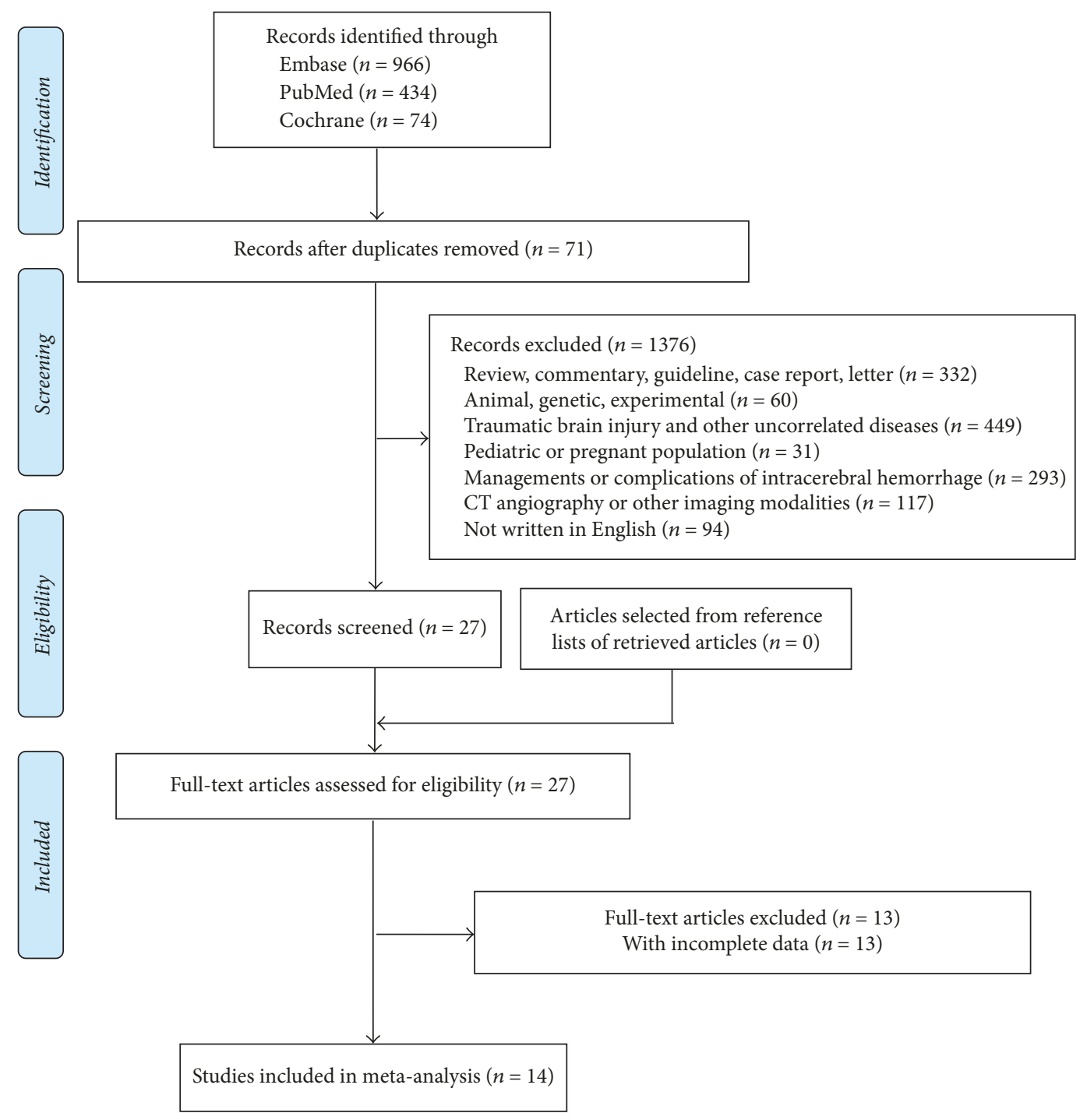

FIGURE 1: The flow diagram of the search process, from [18]. For more information, visit http://www.prisma-statement.org.

heterogeneity signs and $\mathrm{HE}(\mathrm{OR}, 5.17 ; 95 \% \mathrm{CI}, 3.72-7.19, P<$ 0.001 ; Figure 2) with little evidence of heterogeneity $\left(I^{2}=\right.$ $17.6 \%, P=0.30)$.

3.4. Heterogeneity Signs and Poor Outcome. Five studies explored possible associations between heterogeneity signs on NCCT and poor outcomes [15, 16, 19, 22, 26]. Overall, we detected a significant increased incidence of poor outcome among patients with heterogeneity signs (OR, 3.60; 95\% CI, $1.98-6.54, P<0.001$, Figure 3 ) with moderate heterogeneity $\left(I^{2}=65.7 \%, P=0.02\right)$.

3.5. Heterogeneity Signs and Mortality. Six studies were included to evaluate the relationship between NCCT heterogeneity signs and mortality $[11,13,16,19,26,27]$. The pooled result favored a positive association between NCCT heterogeneity signs and mortality (OR, 4.64; 95\% CI, 2.96-7.27, $P<0.001$, Figure 4) with low heterogeneity $\left(I^{2}=36.3 \%\right.$, $P=0.17)$.
3.6. Subgroup Analysis, Sensitivity Analysis, and Publication Bias. Results of subgroup analyses were available in Table III in the online-only Data Supplement. In the analysis of HE and mortality, differences in all subgroups were statistically significant, while no significant results were detected in the subgroups of sample size $<150$ and secondary ICH $(P=0.1)$ when analyzing the relationship between heterogeneity signs and poor outcome. In the sensitivity analysis, no significant result was detected when excluding studies one by one in all analysis. Publication bias was not detected by Egger's test except in analysis examining the relationship between heterogeneity signs and mortality $(P=0.031)$.

\section{Discussion}

Significant HE was a well-established predictor of clinical prognosis and mortality in ICH, and NCCT findings might potentially predict $\mathrm{HE}$ as suggested by previous studies [6$8]$. Our results revealed that heterogeneity signs detected on 


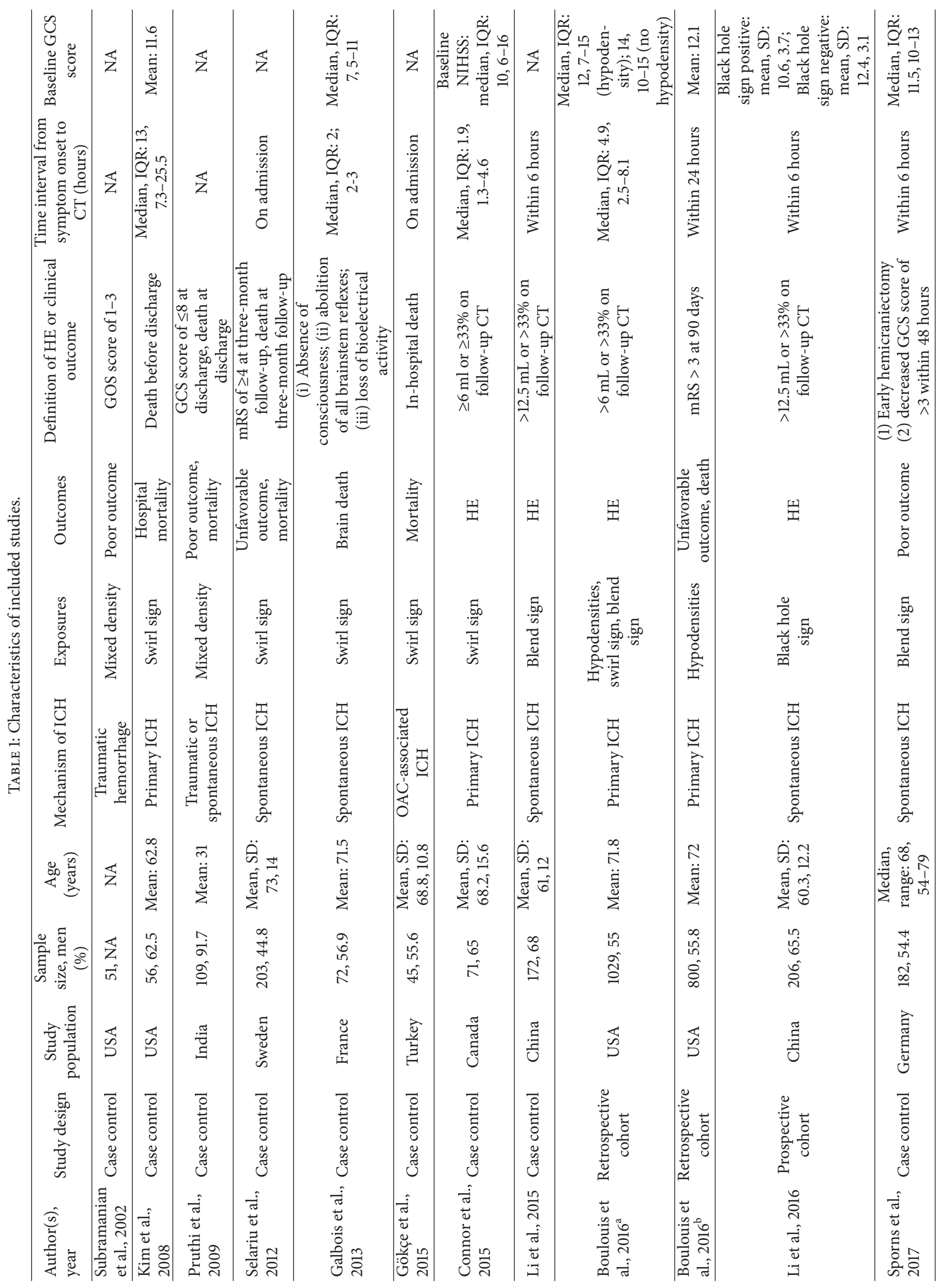




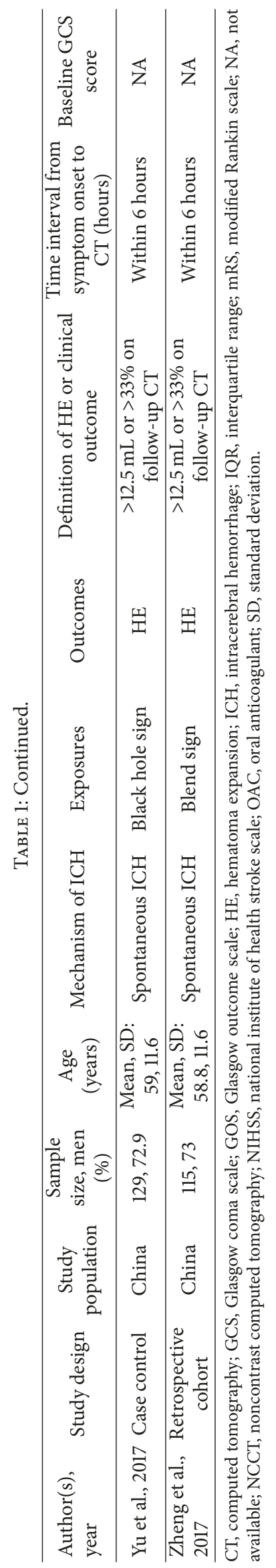




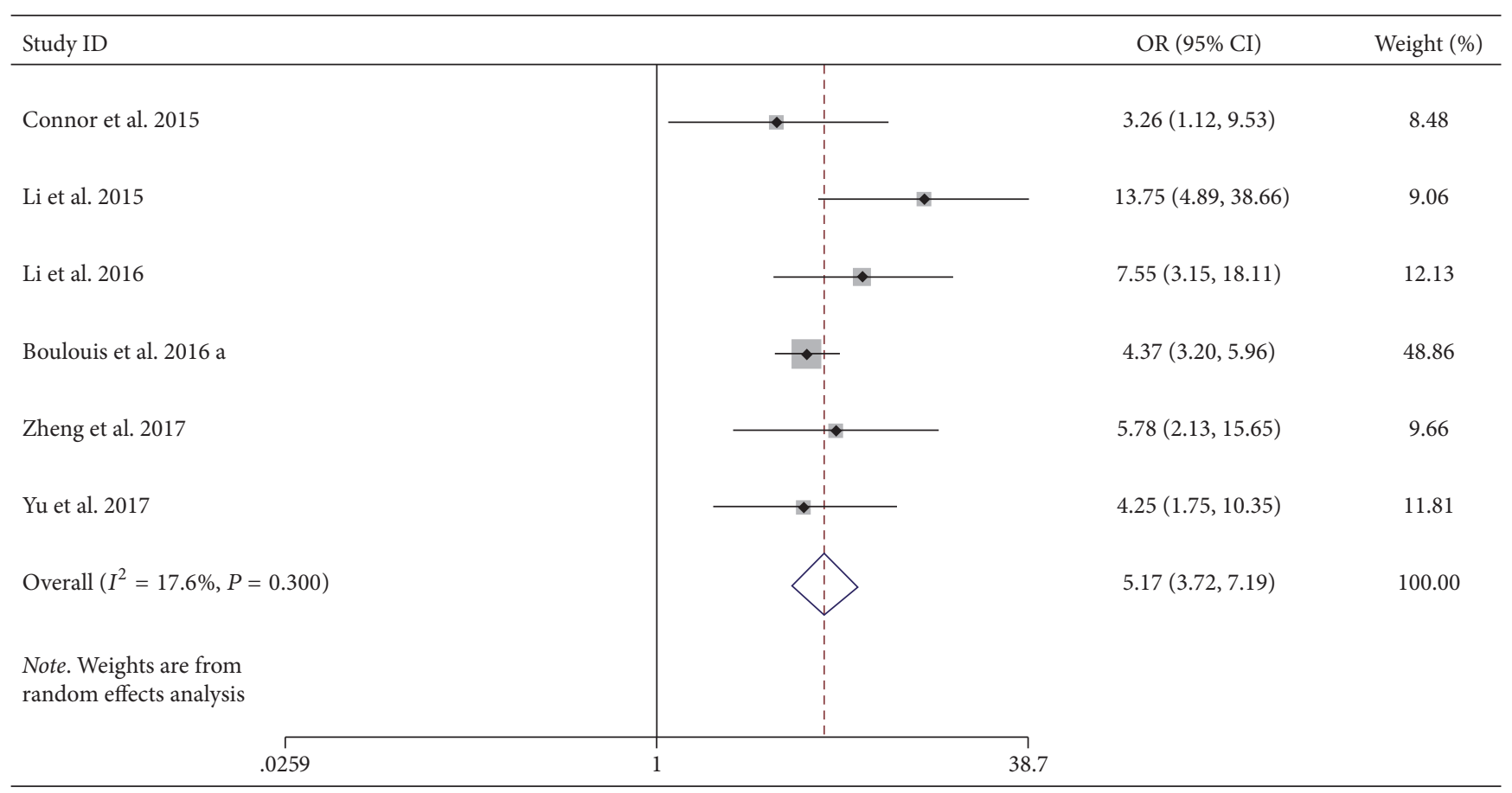

FIGURE 2: Forest plots of NCCT heterogeneity signs and HE. CI, confidence interval; HE, hematoma expansion; NCCT, noncontrast computed tomography; OR, odds ratio.

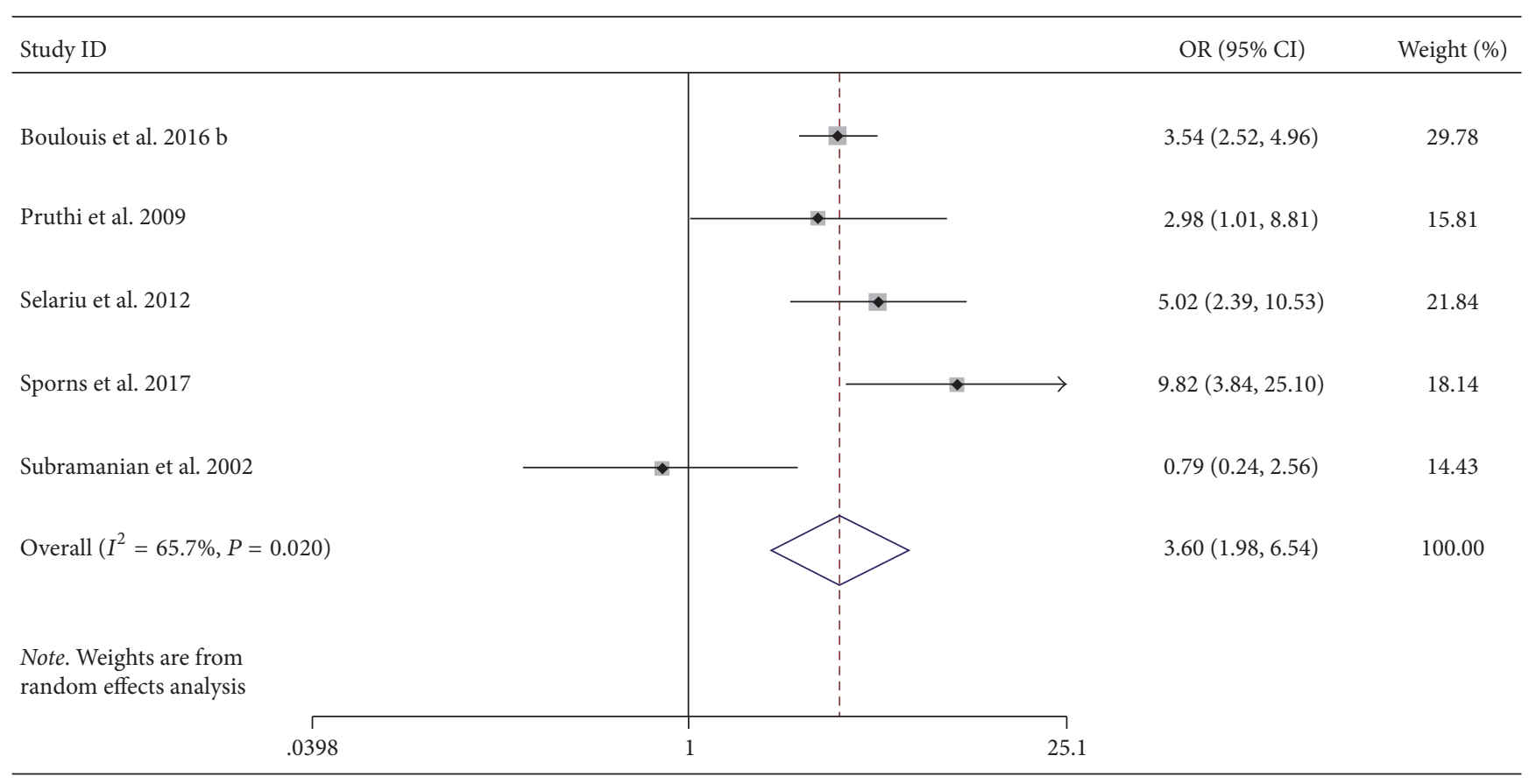

FIGURE 3: Forest plots of NCCT heterogeneity signs and poor outcome. CI, confidence interval; HE, hematoma expansion; NCCT, noncontrast computed tomography; OR, odds ratio.

NCCT were positively associated with the increased risk of HE $(P<0.001)$, poor outcome $(P<0.001)$, and mortality rate $(P<0.001)$, indicating the potential predictive value of heterogeneity signs for $\mathrm{HE}$, poor prognosis, and mortality in ICH patients. In the subgroup analysis of poor outcome, there were no statistically significant differences for the subgroups of sample size $<150$ and secondary ICH, which might imply that small sample size or secondary $\mathrm{ICH}$ would increase the heterogeneity and undermine the predictive value of heterogeneity signs in ICH. 


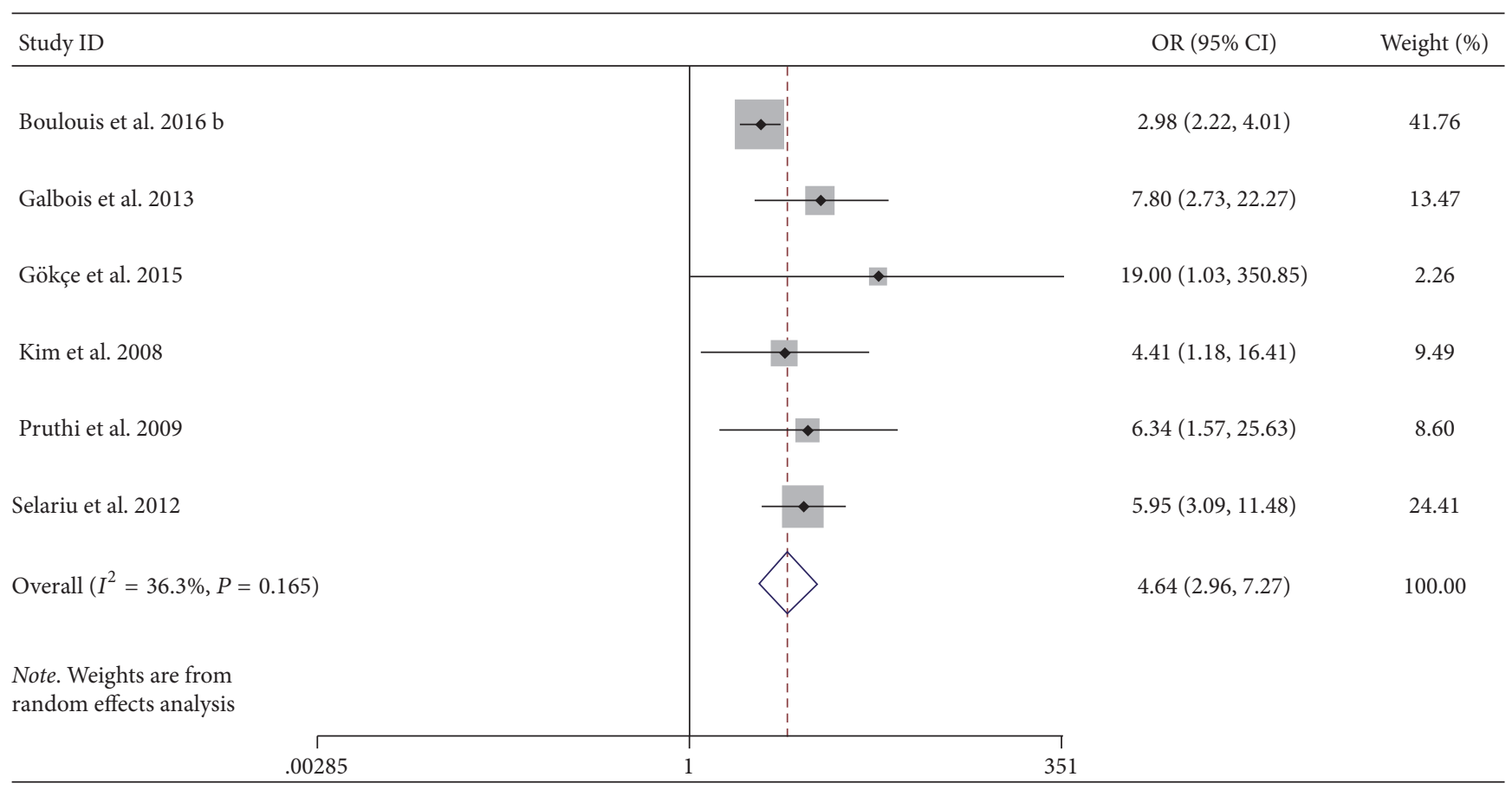

FIGURE 4: Forest plots of NCCT heterogeneity signs and mortality. CI, confidence interval; HE, hematoma expansion; NCCT, noncontrast computed tomography; OR, odds ratio.

The pathophysiology of NCCT heterogeneity remains elusive currently. Hematoma density on NCCT has close relationship with the phase of hemorrhage, number of foci of the blood, and hematocrit [31, 32]. It is suggested that short time interval to NCCT, large hematoma volumes, and anticoagulants are all related to NCCT heterogeneity, as well as to severe clinical presentations and worse prognosis [33]. Pathophysiologically, hypodensities on NCCT seem to indicate blood at an early evolution stage [21, 31]. Liquid blood tends to hypoattenuate on NCCT relative to surrounding structures in acute phase [31]. However, whether hypodensity implies active hemorrhage or just responds to impaired coagulation processes is still unknown [34].

According to our study, heterogeneity signs included a broad spectrum of imaging markers on NCCT, among which the hypodensity [21,35], swirl sign [21, 25], blend sign $[20,21,29,35]$, and black hole sign $[23,28]$ had been investigated previously regarding their relationships with hematoma growth. Hypodensities were proved to be associated with HE in two studies conducted by Boulouis et al. and Morotti et al. [21,35]. Similarly, swirl sign and black hole sign were also indicated to be positively related to $\mathrm{HE}[21,23,25,28]$. However, four studies explored the predictive value of blend sign for HE and their conclusions were different. As for Li et al., Zheng et al., and Morotti et al., they detected that blend sign was a predictive factor for hematoma growth $[20,29,35]$, while this relationship was not statistically significant in the study by Boulouis et al. [21].

Additionally, previous studies explored the relationships of NCCT markers including hypodensities $[26,35]$, mixed density $[15,16]$, swirl sign $[11,13,19,27]$, and blend sign
$[22,35]$ with clinical outcome after ICH. Swirl sign and blend sign were indicated to be statistically associated with clinical outcome such as unfavorable outcome, mortality, or brain death $[11,13,19,22,27,35]$. However, the conclusions were inconsistent concerning the predictive value of hypodensities and mixed density for clinical outcome. Morotti et al. failed to detect a positive connection between hypodensities and unfavorable outcome in the multivariate analysis [35]. Subramanian et al. [15] proved in a case control study involving 51 participants that NCCT mixed density was not correlated with the increased risk of active bleeding or unfavorable outcome. The discrepancies might derive from the inherent interpreter differences in identifying hematoma hypodensity or variability in recording active bleeding. Therefore, the assessment of density alone should not be applied in routine clinical practice and more researches were needed regarding the optimal method of evaluating density or predicting $\mathrm{HE}$ [14].

To our knowledge, this study was the first systematic review and meta-analysis available exploring the relationships between heterogeneity signs on NCCT and the presence of HE. Our results supported that hematoma heterogeneity signs were potential predictors in identifying patients at a high risk of HE. In addition, we investigated the relationships between hematoma heterogeneity signs and prognosis of $\mathrm{ICH}$, and statistically significant results were detected. Our study also had advantage in including trials from different countries and regions which were representative enough. These included studies were all newly published in recent years and the heterogeneity among studies was moderate. 
Several limitations in our study should be noticed. Firstly, relationships between hematoma heterogeneity and $\mathrm{HE}$ or clinical outcome were not examined due to the differences between the definitions of hematoma heterogeneity and NCCT signs in our study. Hematoma heterogeneity was defined according to a 5-point grading scale first introduced and validated by Barras et al. [12], as density categories of no or small heterogeneity ( 1 or 2 ) were labeled homogeneous and categories of progressive heterogeneity (3 to 5) heterogeneous. However, there were overlaps between hematoma heterogeneity and heterogeneity signs, and thus some valuable patient data might be missed in our study. Secondly, there was variability among included studies regarding the definition of heterogeneity signs and HE, which might derive from the different criteria used in different studies. Thirdly, despite moderate heterogeneity in our analysis, different biases such as the selection bias or publication bias did exist owing to the defects of meta-analysis itself. Patients with NCCT heterogeneity signs might have large initial volume or irregular shape of hematoma [12]. The language was confined to English, which might overlook some valuable non-English studies. Notably, we found publication bias in the analysis of mortality, indicating a potentially overestimated association between NCCT heterogeneity signs and mortality, since positive results were more likely to be reported. Fourthly, different indicators with variable definitions were used to evaluate the clinical outcomes in included literatures, which might be the source of heterogeneity among studies [26, 36]. Lastly, the quantity of included studies was limited and most of them were retrospective, without eligible high-quality trials.

\section{Conclusions}

Despite the limitations, our findings raised certain clinical implications that hematoma heterogeneity signs were positively associated with an increased risk of $\mathrm{HE}$ and poor clinical outcome and mortality rate in ICH. However, caution was needed when interpreting our results due to the moderate heterogeneity among studies and limited number of studies in our analysis. Further large scale prospective study was required to confirm these findings in the future.

\section{Conflicts of Interest}

All authors of this manuscript declare that they have no conflicts of interest.

\section{Authors' Contributions}

Danfeng Zhang and Jigang Chen were responsible for the study design and literature research. Qiang Xue, Bingying $\mathrm{Du}$, and $\mathrm{Ya} \mathrm{Li} \mathrm{were} \mathrm{responsible} \mathrm{for} \mathrm{the} \mathrm{statistical} \mathrm{analysis.}$ Tao Chen, Ying Jiang, and Junyu Wang conducted the data interpretation and acquisition. Yan Dong and Lijun Hou prepared the manuscript. Danfeng Zhang, Jigang Chen, Qiang Xue, and Bingying Du contributed equally to this work.

\section{Supplementary Materials}

Supplemental Method. The complete search algorithm for PubMed is as follows. PubMed ${ }^{(}((()((($intracerebral hemorrhage) OR intracranial hemorrhage) OR intracerebral hematoma) OR intracranial hematoma) OR cerebral hemorrhage) OR subdural hemorrhage) OR epidural hemorrhage)) AND (((((hypodensit*) OR density) OR swirl sign) OR blend sign) OR black hole sign))) AND computed tomograph* Supplemental Table I. Categorization, exposure, and original data of included studies. CT, computed tomography; HE, hematoma expansion; HU, Hounsfield unit; ICH, intracerebral hemorrhage; $N$, number. Supplemental Table II. Quality scores of included studies using Newcastle-Ottawa Scale (maximum score of 9). 0 = "No," "Unable to determine," or "Not available". Supplemental Table III. Subgroup analyses of heterogeneity signs on NCCT and risk of HE. CI, confidence interval; HE, hematoma expansion; ICH, intracerebral hemorrhage; NCCT, noncontrast computed tomography; OR, odds ratio. (Supplementary Materials)

\section{References}

[1] J. P. Broderick, T. G. Brott, J. E. Duldner, T. Tomsick, and G. Huster, "Volume of intracerebral hemorrhage: a powerful and easy-to-use predictor of 30-day mortality," Stroke, vol. 24, no. 7, pp. 987-993, 1993.

[2] A. I. Qureshi, S. Tuhrim, J. P. Broderick, H. H. Batjer, H. Hondo, and D. F. Hanley, "Spontaneous intracerebral hemorrhage," The New England Journal of Medicine, vol. 344, no. 19, pp. 1450-1460, 2001.

[3] C. J. van Asch, M. J. Luitse, G. J. Rinkel, I. van der Tweel, A. Algra, and C. J. Klijn, "Incidence, case fatality, and functional outcome of intracerebral haemorrhage over time, according to age, sex, and ethnic origin: a systematic review and metaanalysis," The Lancet Neurology, vol. 9, no. 2, pp. 167-176, 2010.

[4] S. Kazui, H. Naritomi, H. Yamamoto, T. Sawada, and T. Yamaguchi, "Enlargement of spontaneous intracerebral hemorrhage: incidence and time course," Stroke, vol. 27, no. 10, pp. 1783-1787, 1996.

[5] T. Brott, J. Broderick, R. Kothari et al., "Early hemorrhage growth in patients with intracerebral hemorrhage," Stroke, vol. 28, no. 1, pp. 1-5, 1997.

[6] C. Delcourt, Y. Huang, H. Arima et al., "Hematoma growth and outcomes in intracerebral hemorrhage: the INTERACT1 study.," Neurology, vol. 79, no. 4, pp. 314-319, 2012.

[7] S. M. Davis, J. Broderick, M. Hennerici et al., "Hematoma growth is a determinant of mortality and poor outcome after intracerebral hemorrhage," Neurology, vol. 66, no. 8, pp. 11751181, 2006.

[8] J. C. Hemphill III, S. M. Greenberg, C. Anderson et al., "Guidelines for the management of spontaneous intracerebral hemorrhage: a guideline for healthcare professionals from the American Heart Association/American Stroke Association," Stroke, vol. 46, no. 7, pp. 2032-2060, 2015.

[9] F.-Z. Du, R. Jiang, M. Gu, C. He, and J. Guan, “The accuracy of spot sign in predicting hematoma expansion after intracerebral hemorrhage: A systematic review and meta-analysis," PLoS ONE, vol. 9, no. 12, Article ID el15777, 2014. 
[10] A. Del Giudice, D. D’Amico, J. Sobesky, and I. Wellwood, "Accuracy of the spot sign on computed tomography angiography as a predictor of haematoma enlargement after acute spontaneous intracerebral haemorrhage: A systematic review," Cerebrovascular Disease, vol. 37, no. 4, pp. 268-276, 2014.

[11] J. Kim, A. Smith, J. C. Hemphill III et al., "Contrast extravasation on CT predicts mortality in primary intracerebral hemorrhage," American Journal of Neuroradiology, vol. 29, no. 3, pp. 520-525, 2008.

[12] C. D. Barras, B. M. Tress, S. Christensen et al., "Density and shape as CT predictors of intracerebral hemorrhage growth," Stroke, vol. 40, no. 4, pp. 1325-1331, 2009.

[13] A. Galbois, P.-Y. Boëlle, E. Hainque et al., "Prediction of evolution toward brain death upon admission to ICU in comatose patients with spontaneous intracerebral hemorrhage using simple signs," Transplant International, vol. 26, no. 5, pp. 517-526, 2013.

[14] C. Delcourt, S. Zhang, H. Arima et al., "Significance of Hematoma Shape and Density in Intracerebral Hemorrhage: The Intensive Blood Pressure Reduction in Acute Intracerebral Hemorrhage Trial Study," Stroke, vol. 47, no. 5, pp. 1227-1232, 2016.

[15] S. K. Subramanian, M. H. Roszler, B. Gaudy, and D. B. Michael, "Significance of computed tomography mixed density in traumatic extra-axial hemorrhage," Neurological Research, vol. 24, no. 2, pp. 125-128, 2002.

[16] N. Pruthi, A. Balasubramaniam, B. A. Chandramouli et al., "Mixed-density extradural hematomas on computed tomography-prognostic significance," Surgical Neurology, vol. 71, no. 2, pp. 202-206, 2009.

[17] D. Moher, A. Liberati, J. Tetzlaff, and D. G. Altman, "Preferred reporting items for systematic reviews and meta-analyses: the PRISMA statement," British Medical Journal, vol. 339, article b2535, 2009.

[18] D. Moher, A. Liberati, J. Tetzlaff, and D. G. Altman, "Preferred reporting items for systematic reviews and meta-analyses: the PRISMA statement," PLoS Medicine, vol. 6, no. 7, Article ID e1000097, 2009.

[19] E. Selariu, E. Zia, M. Brizzi, and K. Abul-Kasim, "Swirl sign in intracerebral haemorrhage: definition, prevalence, reliability and prognostic value," BMC Neurology, vol. 12, article no. 109, 2012.

[20] Q. Li, G. Zhang, Y.-J. Huang et al., "Blend Sign on Computed Tomography: Novel and Reliable Predictor for Early Hematoma Growth in Patients With Intracerebral Hemorrhage," Stroke, vol. 46, no. 8, pp. 2119-2123, 2015.

[21] G. Boulouis, A. Morotti, H. Bart Brouwers et al., "Association between hypodensities detected by computed tomography and hematoma expansion in patients with intracerebral hemorrhage," JAMA Neurology, vol. 73, no. 8, pp. 961-968, 2016.

[22] P. B. Sporns, M. Schwake, R. Schmidt et al., "Computed tomographic blend sign is associated with computed tomographic angiography spot sign and predicts secondary neurological deterioration after intracerebral hemorrhage," Stroke, vol. 48, no. 1, pp. 131-135, 2017.

[23] Q. Li, G. Zhang, X. Xiong et al., "Black hole sign: Novel imaging marker that predicts hematoma growth in patients with intracerebral hemorrhage," Stroke, vol. 47, no. 7, pp. 1777-1781, 2016.

[24] J. P. T. Higgins, S. G. Thompson, J. J. Deeks, and D. G. Altman, "Measuring inconsistency in meta-analyses," British Medical Journal, vol. 327, no. 7414, pp. 557-560, 2003.
[25] D. Connor, T. J. Huynh, A. M. Demchuk et al., "Swirls and spots: relationship between qualitative and quantitative hematoma heterogeneity, hematoma expansion, and the spot sign," Neurovascular Imaging, vol. 1, no. 1, 2015.

[26] G. Boulouis, A. Morotti, H. B. Brouwers et al., "Noncontrast Computed Tomography Hypodensities Predict Poor Outcome in Intracerebral Hemorrhage Patients," Stroke, vol. 47, no. 10, pp. 2511-2516, 2016.

[27] E. Gökçe, M. Beyhan, and B. Acu, "Evaluation of Oral Anticoagulant-Associated Intracranial Parenchymal Hematomas Using CT Findings," Clinical Neuroradiology, vol. 25, no. 2, pp. 151-159, 2015.

[28] Z. Yu, J. Zheng, L. Ma et al., “The predictive accuracy of the black hole sign and the spot sign for hematoma expansion in patients with spontaneous intracerebral hemorrhage," Neurological Sciences, vol. 38, no. 9, pp. 1591-1597, 2017.

[29] J. Zheng, Z. Yu, Z. Xu et al., “The Accuracy of the Spot Sign and the Blend Sign for Predicting Hematoma Expansion in Patients with Spontaneous Intracerebral Hemorrhage," Medical Science Monitor International Medical Journal of Experimental \& Clinical Research, vol. 23, p. 2250, 2017.

[30] Z. Yu, J. Zheng, Z. Xu et al., "Accuracy of Shape Irregularity and Density Heterogeneity on Noncontrast Computed Tomography for Predicting Hematoma Expansion in Spontaneous Intracerebral Hemorrhage: A Systematic Review and Meta-Analysis," World Neurosurgery, vol. 108, pp. 347-355, 2017.

[31] P. F. J. New and S. Aronow, "Attenuation measurements of whole blood and blood fractions in computed tomography," Radiology, vol. 121, no. 3 I, pp. 635-640, 1976.

[32] J. N. Pierce, K. H. Taber, and L. A. Hayman, "Acute intracranial hemorrhage secondary to thrombocytopenia: CT appearances unaffected by absence of clot retraction," American Journal of Neuroradiology, vol. 15, no. 2, pp. 213-215, 1994.

[33] A. M. Demchuk, D. Dowlatshahi, D. Rodriguez-Luna et al., "Prediction of haematoma growth and outcome in patients with intracerebral haemorrhage using the CT-angiography spot sign (PREDICT): A prospective observational study," The Lancet Neurology, vol. 11, no. 4, pp. 307-314, 2012.

[34] D. Blacquiere, A. M. Demchuk, M. Al-Hazzaa et al., "Intracerebral Hematoma Morphologic Appearance on Noncontrast Computed Tomography Predicts Significant Hematoma Expansion," Stroke, vol. 46, no. 11, pp. 3111-3116, 2015.

[35] A. Morotti, G. Boulouis, J. M. Romero et al., "Blood pressure reduction and noncontrast $\mathrm{CT}$ markers of intracerebral hemorrhage expansion," Neurology, vol. 89, no. 6, pp. 548-554, 2017.

[36] X. Yao, Y. Xu, E. Siwila-Sackman, B. Wu, and M. Selim, "The HEP Score: A Nomogram-Derived Hematoma Expansion Prediction Scale," Neurocritical Care, vol. 23, no. 2, pp. 179-187, 2015. 


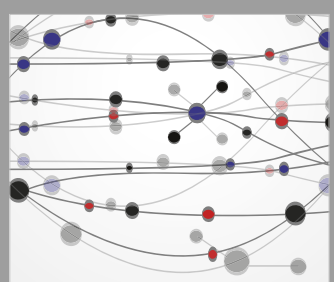

The Scientific World Journal
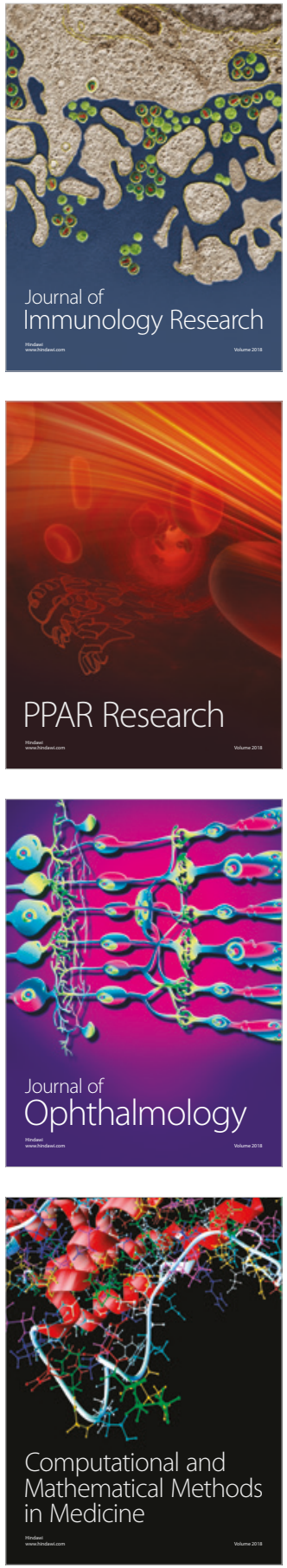

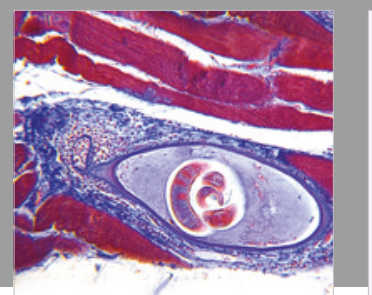

Gastroenterology Research and Practice

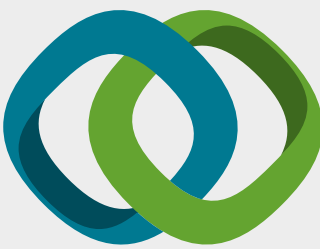

\section{Hindawi}

Submit your manuscripts at

www.hindawi.com
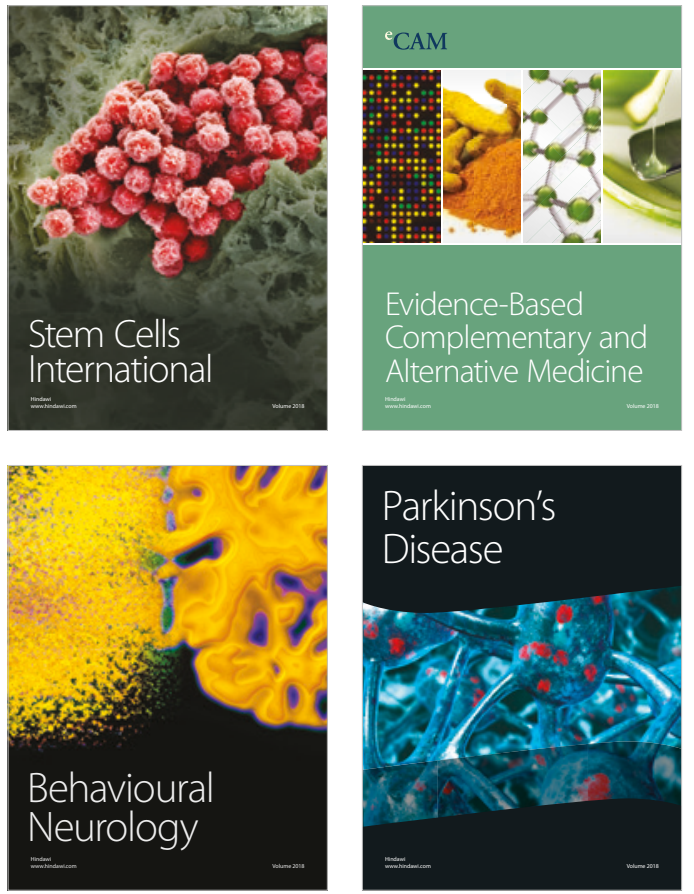

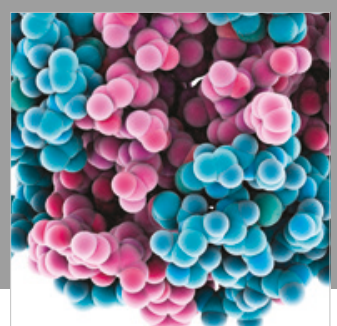

ournal of

Diabetes Research

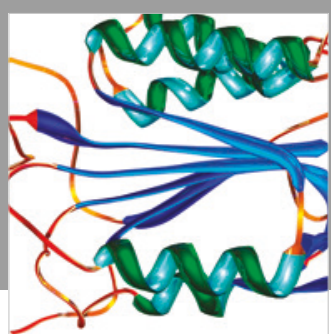

Disease Markers
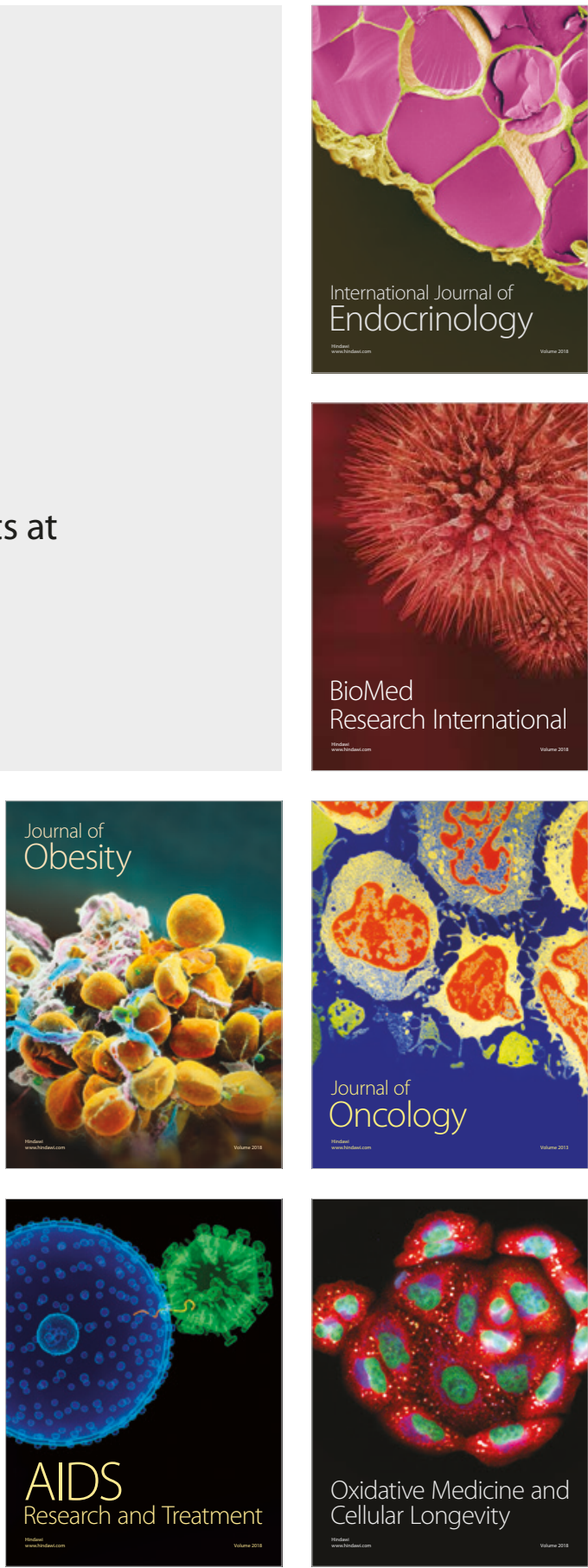